\title{
Electroencephalographic Study in Clinical Cases of Head Injuries.
}

\author{
By \\ Makoto Hayashi. \\ (林 \\ 誠)
}

(From Prof. Katsura's Surgical Clinic Faculty of Medicine, The Tohoku University, Sendai.)

(Received for publication, July 28, 1949)

The electroencephalographic studies in clinical cases of head injuries have been done by Case, T. J., P.C. Bucy ${ }^{11}$, Jasper, Kershman and Elvidge?' (1940), but they gave little attention to the beta waves.

This study will clarify the important function of the beta waves, as well as other abnormal waves, in making an electroencephalographic diagnosis of brain injury.

\section{Material.}

59 cases are included in this survey, which are composed of 37 cases in fresh and old head injuries, and 22 cases in brain operations regarded as brain injuries. (In brain operations, as the conditions of the brain damage had been known clearly, it was expected that they would contribute to clarify the character of the EEG.)

\section{Technic.}

The electroencephalographic tracing used in this survey is made by a four-tube amplifier, the frequency character of which is constant to all frequencies between one and thirty five cycles. In order to do monopolar recording, one silver electrode as a negative pole was attached to the lobe of each ear and another as a positive pole was fastened to the surface of the cleansed scalp of the patient, with saline starch. The latter is movable on the injured regions and their circumferences, left and right forehead, motor area and occiput, according to the place selected for getting the record. The tracing time was usually 20-30 seconds for each area, but was prolonged in case of necessity.

The conditions of recordings are standardized as nearly as possible. The patients are placed on an electrically shielded bed in a darkened room. 
They are instructed to lie as quietly as possible, with the eyes closed, but to remain in a waking state.

\section{Observation of EEG.}

The EEG are studied from the next two points:

1. The average frequency and amplitude of 100 consecutive waves.

2. The histogram of all 100 waves. (The base line fluctuation and the large waves, on which the smaller excursions superimposed, are included in 100-waves.)

Results and Commentary.

I. Cases of head injuries

A. Fresh cases:

In evaluating the EEG of fresh cases, one should distinguish the unconscious cases from the conscious cases, because in the former cases the appearance of delta waves may be considered similar to the natural delta waves in sleep.

a. The unconscious cases are 3 and introduced as follows:

Case 1; A woman aged 24, was knocked down by a jeep and struck her occiput on the pavement. She was unconscious for 10 minutes. The EEG were taken on the following day, when she was still in the chaotic state of consciousness, vomiting several times. The record gained from every region of the head showed the generalized delta waves in frequency from 3.2 to 8 cycles per second, mixed with less beta waves and alpha waves, whereas on the occiput, which seemed to be injured, the beta waves in frequency from 20 to 32 cycles increased. Lumber puncture showed that the spinal fluid was bloody and under a pressure of $450 \mathrm{~mm}$. of water. In a few days she had recovered from the chaotic state of consciousness. (Fig. 1)

Case 2; A man aged 32, was struck by a tramcar, wounded in the face and the occiput, and lost consciousness immediately. The EEG were taken on the following day, when he was still in the chaotic state of consciousness, vomiting several times. The delta waves of high amplitude in frequency from 2 to 8 cycles per second were generalized in the record, and on the right occiput the beta waves in frequency from 12.5 to 32 cycles per second appeared.

Judging from the record, the right occiput was considered to be injured. Lumbar puncture showed that the spinal fluid was bloody and under a pressure of $330 \mathrm{~mm}$. of water. He recovered after 8 days from the chaotic state of consciousness. The EEG was similar to that of cerebral bleeding in the animal experiment, therefore the case was suspected as a 

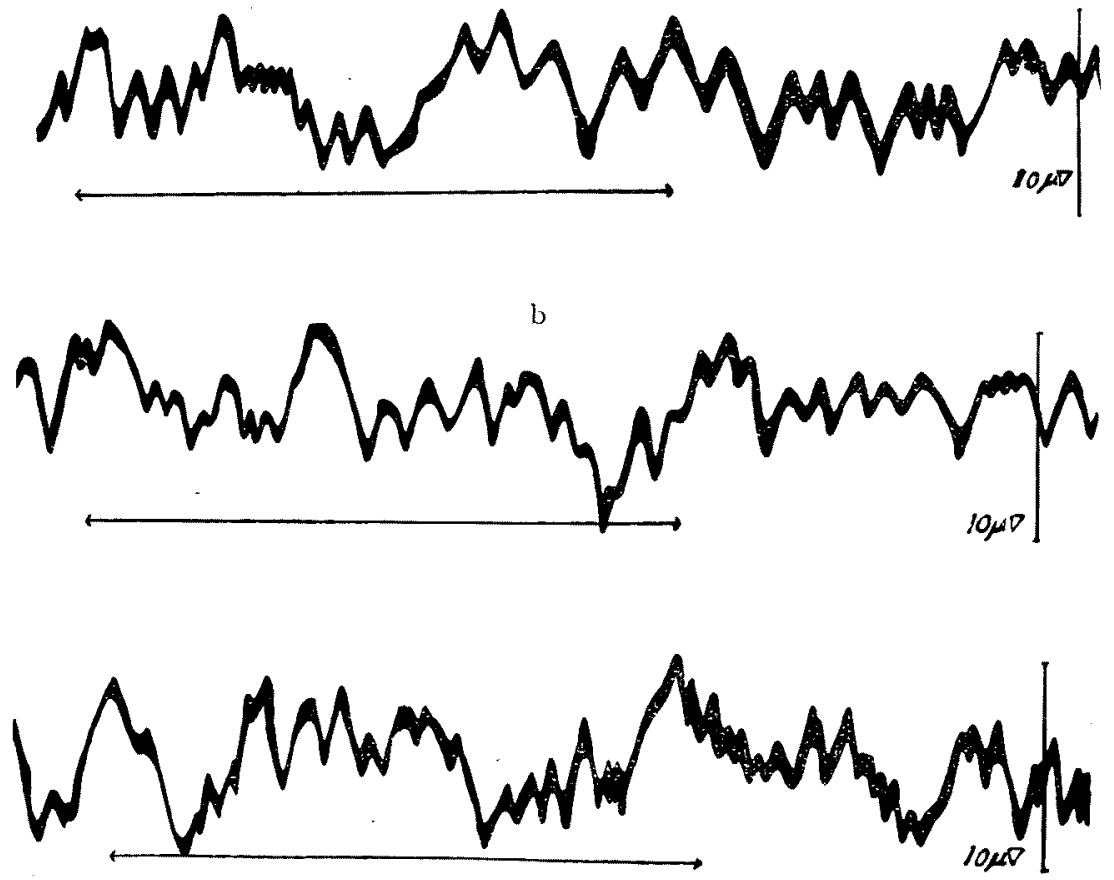

d

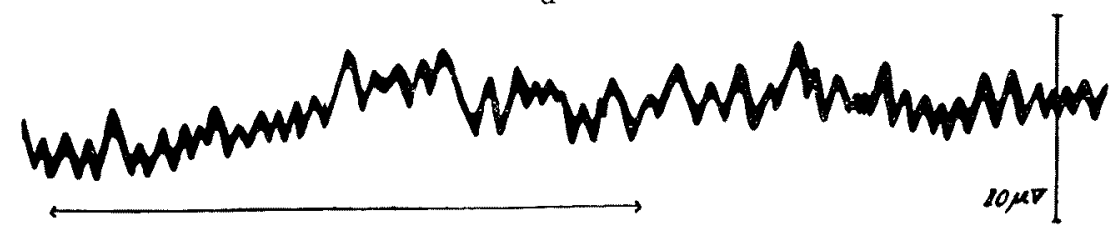

Fig. 1. Case with Unconsciousness
a. Motor Area (left)
b. Motor Area (right)
c. Occiput (left)
d. Occiput (right) (Injured Side) .

subarachnoid hemorrhage. 25 days later the EEG was normal on every area.

Case 3; A man aged 38, was thrown off a tramcar and lost consciousness immediately. When the EEG were taken two days after the injury, he was comatose and sometimes irritable. Lumber puncture showed that the spinal fluid was bloody and under a pressure of $150 \mathrm{~mm}$. of water (in lying position). The record showed the generalized delta waves in frequency from 3.2 to 8 cycles per second and sometimes they were sharp 
and of high potentials. The electrogram was repeated a week after the injury, when the patient was drowsy and sometimes irritable, and of retrograde amnesia in the chaotic state of consciousness. In the latter record, the delta waves were somewhat fast in frequency from 5 to 8 cycles and the alpha waves reappeared.

Especially on the right occiput the EEG were of high amplitude and the beta waves increased. 10 days after, the patient recovered consciousness, but the paralysis of $\mathrm{N}$. oculomotor remained.

This fact indicated that in unconscious cases the delta waves generally appear on the every area and in addition the beta waves increase on the injured region.

b. The cases without the unconsciousness are 19 and introduced as follows:

1. 11 of them showed the normal EEG. All of them had minor head injuries caused by falls, blows and thrown stones. 6 of them lost consciousness, but recovered soon after.

Case 1; (This case is a relatively serious one.)

A woman aged 23, was thrown off a slowlymoving train and knocked her occiput against the concrete of the platform and remained unconscious for 20 minutes. Because of the bleeding from the right ear and the nose and the existing blood in spinal fluid, it was suspected to be a skull base fracture. But the EEG were apparently normal after one week, so that her diagnosis was determined as a subarachnoid hemorrhage.

2. 8 of them showed the abnormal EEG.

(i) 4 cases of them showed the beta waves on the injured region.

Case 1; A boy aged 13, was struck by a truck and knocked his right parietal area against the concrete pavement. The EEG were taken 3 hours later. The beta waves increased on the injured region, the forehead and the occiput, but disappeared 6 days after.

Case 2; A man aged 25, was beastly drunk and knocked his temporal region against the pavement. He was unconscious for a little while and vomited. The headache was specially heavy on the right hemisphere and there was recognized anisocoria $(r .>1$.$) . Spinal fluid was bloody$ and under a pressure of $200 \mathrm{~mm}$. of water (in lying position). The EEG were taken 3 days later. The beta waves recorded on the EEG were of low potentials on the injured region and were lower on the opposite region. The records from the other regions were normal.

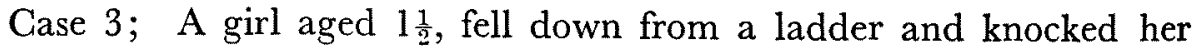
right temporal region and had a depressed fracture on that region. The records were taken a week later and showed the beta waves of relative high potentials on the injured region and the delta waves on the opposite region. 
However, in this case one should consider the age of the patient. According to the studies of Bernhard and Skoglund ${ }^{3}$ (1939), the average number of period of the baby aged from 1 to 2 years is $6.5 \pm 0.1$ cycles. In this case the average period on the opposite region is 6.8 cycles, so that the EEG are to be noted as normal.

Case 4; A man aged 59, knocked his occiput against the ice and was unconscious for 30 minutes. He walked zig-zag like a drunkard and came to this hospital, complaining of a headache of the occiput and of nausea. Nistagmus was recognized on the right eye and Romberg's reaction was positive. Owing to these symptoms, the injury was considered to be in the right cerebellum. On the records taken 6 days after, the beta waves increased on every area of the head, but specially on the occiput.

(ii) 2 cases showed the delta waves on the injured region.

Case 1; A boy aged 9, was knocked on his left forehead by a bat. The wound and the depressed fracture were recognized on the forehead, but there was no history of unconsciousness. The spinal fluid was bloody and its pressure was high. The record taken 3 hours after showed that the period of the EEG was slower on the injured region than on the other regions.

Case 2; A boy aged 7, was struck by a firecar and was wounded in the parietal region. He was unconscious for 16 hours. The records made 4 days after showed that the delta waves of high and slow potentials appeared more frequently on the right hemisphere than on the left, and they were intensified clearly on the injured region.

(iii) 2 cases showed the delta and beta waves on the injured region.

Case 1; A woman aged 27, was struck by a truck and knocked her head against the pavement. She was brought to the hospital in a state of unconsciousness. She had the hematomas and the depressed fractures on both sides of the temporal regions. She recovered her consciousness 15 hours after the injury, but complained of headache, especially on the injured region, vomited several times. The left pupil was larger than the right. The spinal fluid was bloody. The EEG were taken 3 days after, in drowsy condition. The records showed that on the right temporal region the delta waves were of high and slow potentials with the alpha and the beta waves superimposed on them, and on the left temporal region the beta waves of low potentials increased in the presence of a few delta waves.

Case 2; A man aged 29, was strongly struck by a great block of lumber on the forehead. He lost consciousness, but recovered it gradually. And 3 hours after when the EEG were taken it was almost completely recovered. He got the severe wound on the forehead with a depressed 
fracture. The records showed that on the injured region the delta waves were of high and slow potentials with the increased beta and the few alpha waves superimposed on them. And on the left motor area the beta waves were prevalent. On the left occiput some beta waves appeared. On the contrary, on the right motor and occiput the EEG were almost normal. In this case, it was proved through the operation that there was the remarkable destruction of the frontal lobe without the traumatic rupture

a
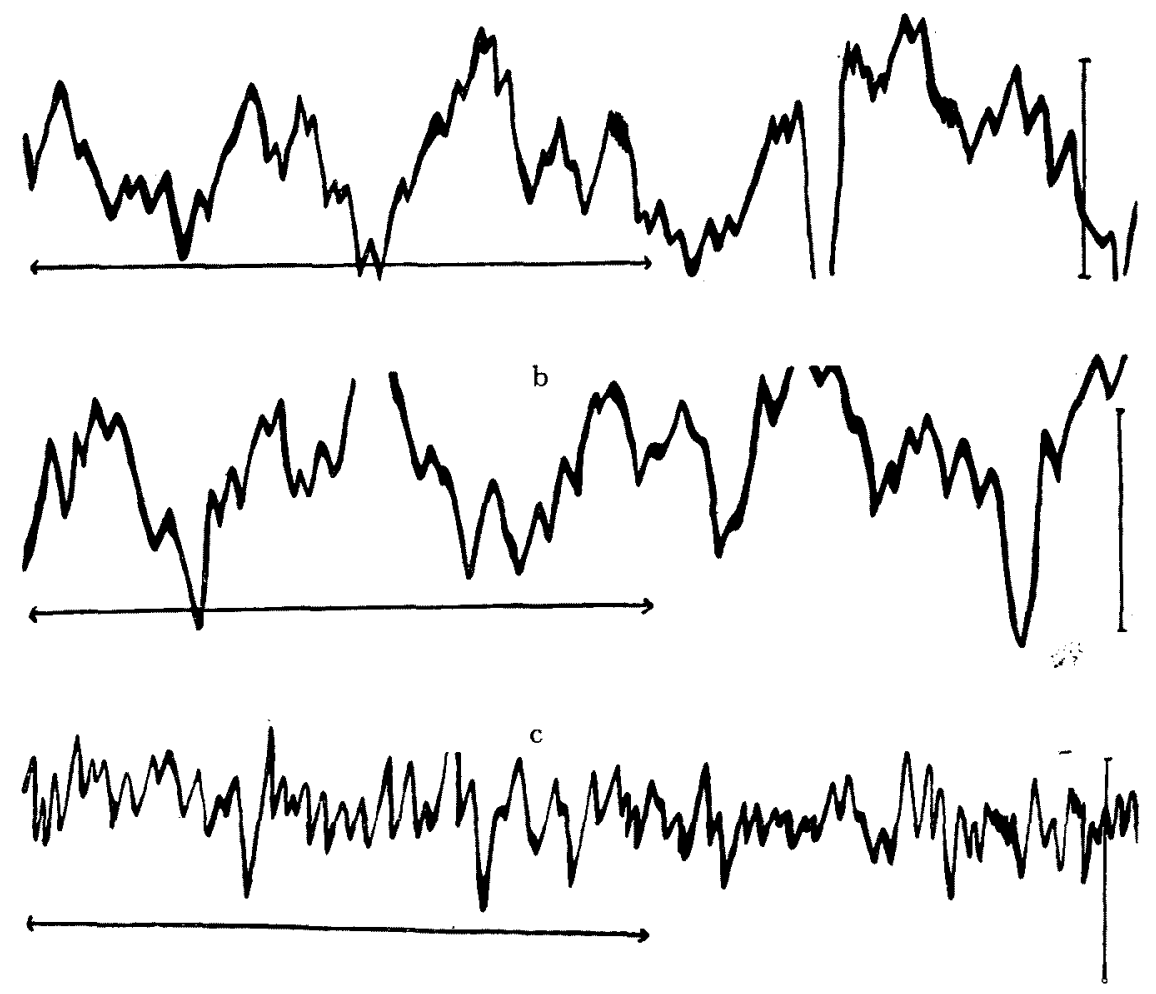

d

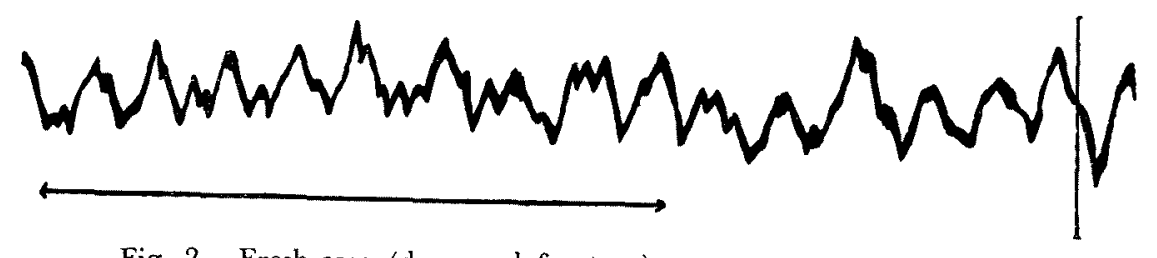

Fig. 2. Fresh case (depressed fracture)

a. Left side of the forehead

b. Injured region

c. Left side of the occiput

d. Right side of the occiput. 
of the dura. (Fig. 2)

Thus, from the information of the EEG of the above mentioned cases, it can be concluded as follows:

1. In cases of losing consciousness, the regular and generalized delta waves are present on each area of the head and the beta waves appear on the injured regions.

2. In cases of no disturbance of consciousness, the EEG are normal when the brain is not damaged.

3. When there are mild brain injuries or cerebral bleeding, the beta waves appear on that area, but in serious brain injuries the delta waves appear with or without the beta waves superimposed on them.

\section{B. Old cases:}

Records were made about 15 cases in which the injuries were from 18 days to 17 years old. 7 of them had epileptic fits.

The abnormalities of the waves from the EEG of the epileptic patients are absolutely characteristic, so that they must be distinguished from other cases.

a. Cases without epileptic fit (8 cases):

1. In 4 cases the EEG are normal.

Case 1; Two of them middle-aged women, who came to the hospital complaining of severe headache caused by old light head injuries. The records of the one were made 5 months after the injury, and those of the other 3 years after. They may be supposed to be suffering from the posttraumatic neurosis.

Case 2; A boy aged 14, was strongly struck in his forehead with a bat and got a depressed fracture of the frontal bone. Temporarily he was unconscious. The records, made 17 and 48 days after the injury, were normal.

Case 3; A boy aged 7, was knocked down by a horse getting an open depressed skull bone fracture and the brain prolapse. In spite of the history of the brain prolapse, the EEG, taken 59 days after the injury, were not abnormal.

2. In 3 cases the EEG were characterized with the beta waves on the injured region.

Case 1; A man aged 20, knocked his forehead in a railway accident getting a wound and a depressed fracture of the skull as large as an egg. He was unconscious for 7 days. He complained of the left diplopia caused by the depression of the left eyeball. The records, made $3 \frac{1}{2}$ months after the injury, showed that the beta waves were present on each area of the 


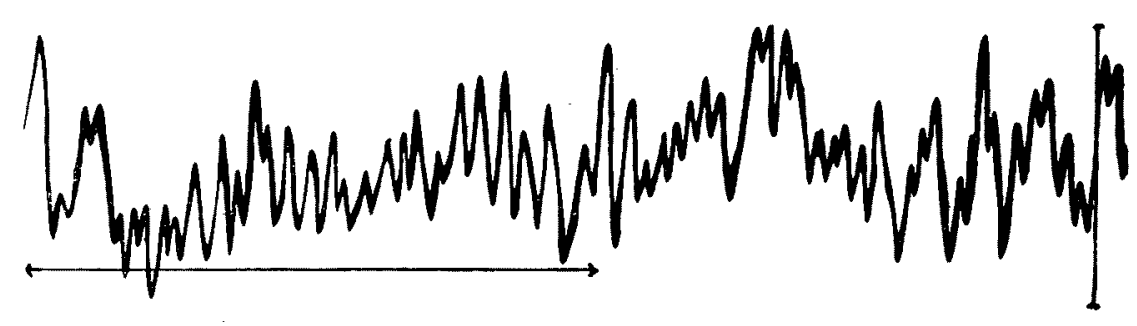

$\mathrm{b}$
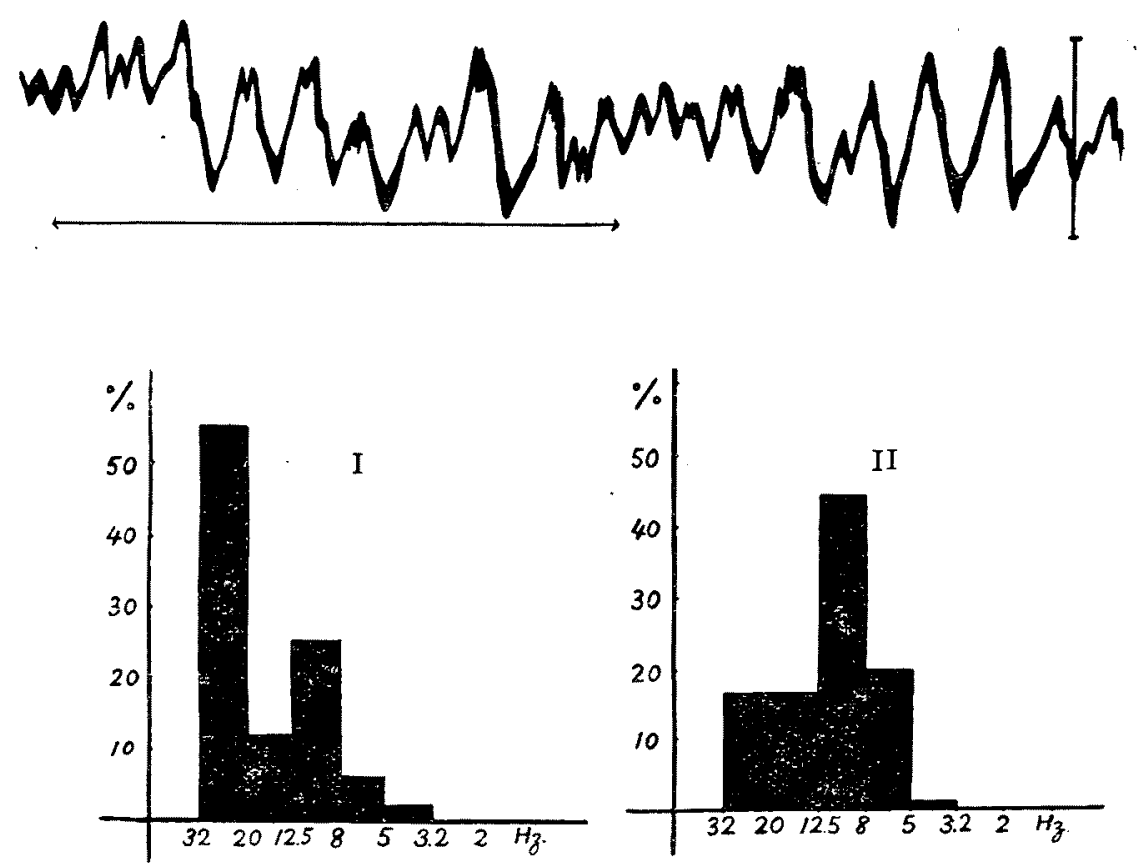

Fig. 3. Old case (contusio cerebri)
a. Injured region (left side of the forehead)
b. Corresponding region in the opposite hemisphere
c. Histogram

I. Injured region

II. Opposite region.

left hemisphere, especially remarkably on the injured region. (Fig. 3)

Case 2; A man aged 22, was struck by a transformer falling down and got an open wound, and a fracture of the skull. On the records, made 3 weeks after, the beta waves increased apparently on the injured region.

Case 3; A man aged 63, was struck on his left occiput by a motorcar, getting a tumor as large as a clenched fist. He was unconscious for 10 
minutes. He had a pain in the occiput about 4 months after. On the records made 6 months after, the beta waves increased a few on the injured region.

Judging from these cases, it may be summarized that the beta waves remain in the injured regions for a considerably long time, when the brain tissues are destroyed.

3. In the following case, the EEG are somewhat distinguishable.

Case 1; A man aged 47, was wounded in a railway accident, and got a depressed fracture of the left temporal bone and the comminuted fracture of the skull extending from parietal to fronto-temporal region. Consciousness was recovered 7 days after. He had a left facial paralysis, hypesthesia of the right side of the body and motor aphasia. On the records about 4 months after the epileptiform waves, such as spike and seizure waves, were recognized on the under part of the right motor area and the occiput. 1 year after the injury these epileptiform waves disappeared.

b. Cases with epileptic fits:

There are 7 cases in which the epileptic fits happened after the head injuries. The records were made from 2 years to 17 years after the injuries. And the EEG can be classified as follows:

1. In 4 cases the EEG were normal.

2. In 2 cases the delta waves were generalized and the beta waves increased on the injured regions.

3. In one case the delta waves äppeared on the injured region.

To Summarize:

1. The EEG were normal when there was no destruction of the brain tissues in old cases, just as in fresh cases.

2. When the brain tissues were destroyed, the beta waves on the injured regions used to remain for a considerably long time in all cases except one.

Through all cases, the epileptic fits were indifferent to the abnormal waves. In one case without the epileptic fit, in which the epileptiform waves appeared, it is an interesting problem whether epileptic fits would follow in the future or not.

\section{EEG after brain operations.}

22 brain operations were sorted as follows:
A. Skull bone tumor
1 .
B. Brain tumor
8.
C. Lobotomy
5.
D. Epilepsy
8. 
A. In the case of skull bone tumor the EEG were normal 13 days after the tumor exstirpation.

B. In 8 cases of brain tumors the EEG were as follows:

1. In 3 cases of hypophysial tumors, the records made from 20 to 40 days after the operation showed that the beta waves increased noticeably on the operation wounds. (The frontal lobes were not resected.)

2. In case of separation of the optic nerves, in which the frontal lobe was not resected, but was pressed to the skull bone for a long time, the records made 6 days after the operation showed on the operation wound the appearance of the delta waves which disappeared 15 days after.

3 . In 3 cases ( 2 hypophysis adenoma, 1 meningioma) in which the EEG were taken a relative long time (from $\frac{1}{2}$ to 7 years) after the tumor resections, the records were fairly normal.

4. The case, which had a tumor in the ventricle, fell into a coma after the operation and its EEG were almost abolished. It may be associated with existence of severe brainsteam damage. In this case the patient died in a short time. (Fig. 4)

C. Lobotomy:

1. In 3 of 5 cases of the lobotomy the records showed temporarily after the operation around the operation wound the appearance of the delta waves which disappeared about 3 weeks after.

2. In 2 cases of them, the beta waves increased on the operation wound and they were free from the delta waves or superimposed on them.

According to the EEG in process of the wound recovery after the operations (brain tumor resection and lobotomy), it may be considered as follows: (Fig. 5 and 6)

1. The delta waves appear temporarily (for from 7 to 20 days), when the brain tissues are destroyed.

2. The beta waves increase apparently after the disappearance of the delta waves and a most prevail from 20 to 30 days.

D. Epilepsy (3 traumatic and 5 idiopathic):

a. Traumatic epilepsy:

Case 1; A girl aged 16, fell down 50 days after her birth and got a great cystoma reaching the subcutane through the skull bone on the left occipital lobe, of which the brain tissue almost degenerated. Before the operation the EEG showed the beta waves of low potentials on that region. After the cicatrix of the cerebral meninges and the degenerated brain tissue were resected and the broad aponeurosis was implanted on that region, the epileptic fit entirely disappeared. But the records made 36 days after the operation were similar to those before it.

Case 2; A woman aged 19, was injured by a nail in the left side of 


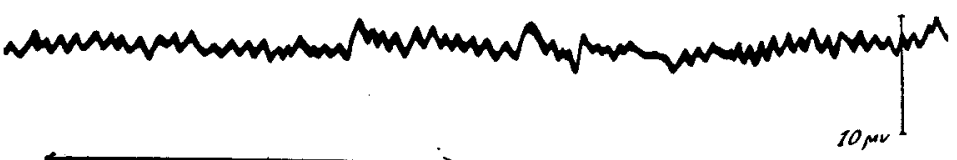

Fig. 4. Collaps after the brain injury.

a

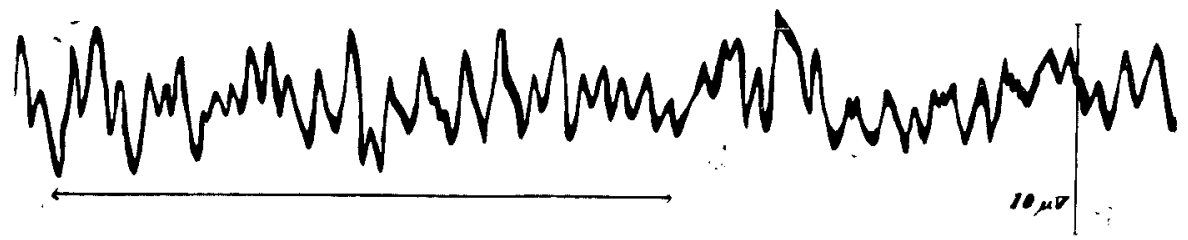

b

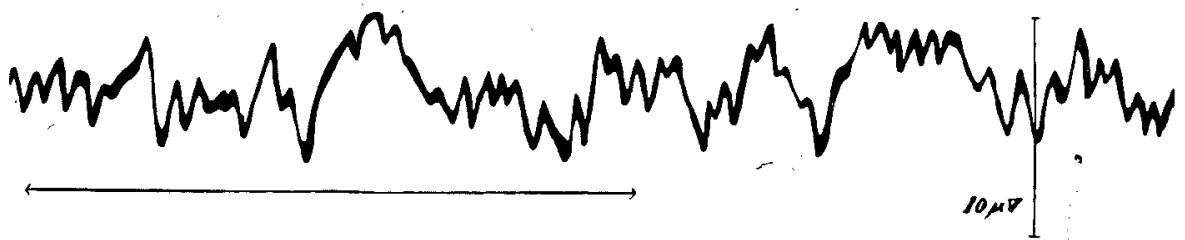

c

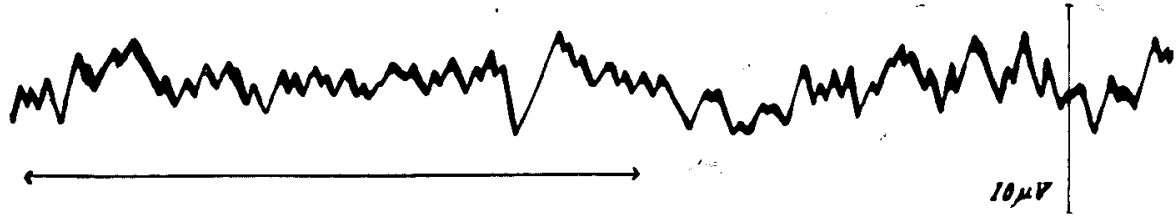

d

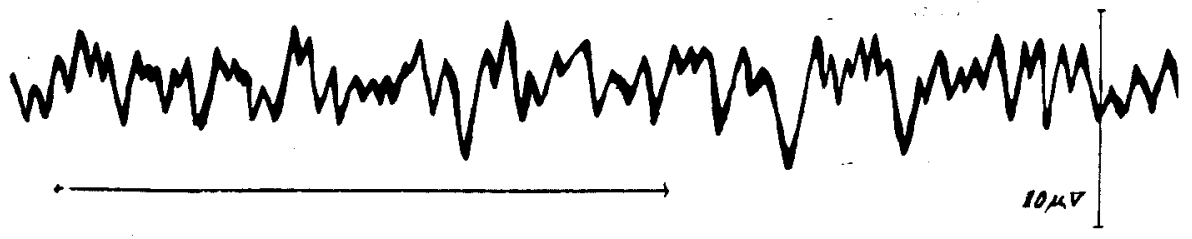

Fig. 5. Process of the wound recovery after the operation
a. Before operation
b. The 6 th day after the operation -
c. The 15 th day after the operation
d. The 43 rd day after the operation. 


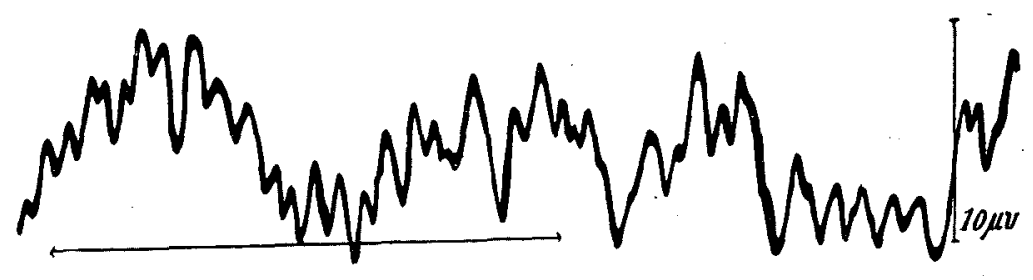

b

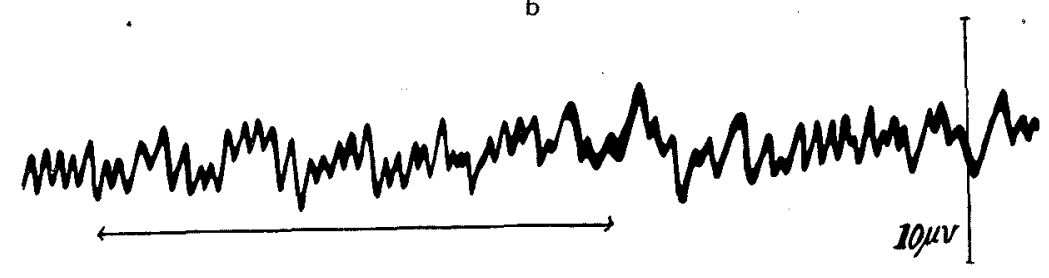

c

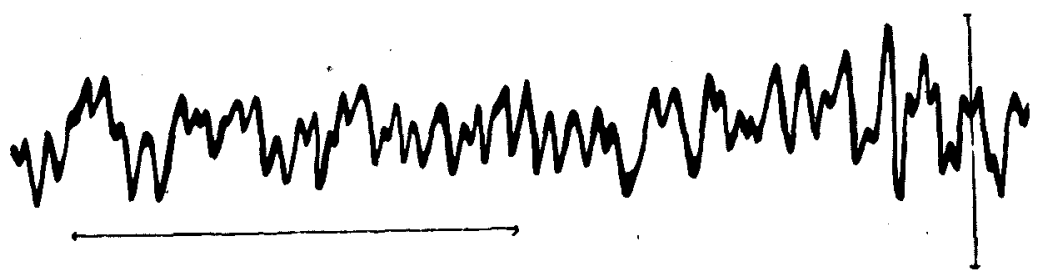

Fig. 6. Process after the lobotomy on the injured region
a. The 7 th day after the lobotomy
b. The 20th day after the lobotomy
c. The 40th day after the lobotomy.

her head at the age of 1 year. The motor paralysis of the right upper limb occurred and epileptic fits began at the age of about 15 years. The brain-durascar was resected by the operation. The records made 9 days after the operation showed the beta waves of low amplitude on the wound.

Case 3; A man aged 23, was a patient of traumatic epilepsy. 5 days. after the operation, the generalized delta waves appeared. On the wound the EEG showed the delta waves of remarkably high potentials with the alpha and the beta waves superimposed on them.

b. Idiopathic epilepsy:

In 4 cases of 5 idiopathic epilepsy, the resections of the $6 \alpha \beta$ (Vogt) were done and in one case the lobotomy was attempted. In all of them the appearance of the delta waves were recognized on the operation wound. 
In evaluating the change in the course of time of the EEG on the operation wound, it was noted that when the delta or the spike waves were observed from 12 to 15 days after the operation on the wound, the former of high amplitude reappeared from 20 to 32 days after on the same place and sometimes epileptiform activity co-existed.

It is most interesting that in the epileptic cases the delta waves remained for a long time on the wound, in contrast to the non epileptic cases.

\section{General Conclusion.}

The principal results in the above mentioned clinical cases of head injuries may be summarized as follows:

1. Minor head injuries with no brain substantial damage, either fresh or old, produce no changes in the electrical cortical activity in the injured regions. But there is one exception, in which the part of brain prolapse was resected completely to be cured and the EEG was normal several months after the accident.

2. The beta waves observed on the injured regions in cases of the fresh head injuries mean the relative minor brain injuries, but in cases of relative major brain injuries they are seen on both the injured and the distant regions. In these cases on the decrease in the course of the recovery of the wounds, and the beta waves take the place of them in about a week, remaining relatively for a long time.

These temporary delta waves on the injured regions may be associated with the cerebral edema. According to the experimentation on cerebral edema made by Zeniya," member of our institute, the records showed the delta waves remaining from several days to $1-2$ weeks.

3. The delta waves observed on the injured region in cases of the fresh head injuries mean the major brain injuries, and the frequency and the degree of the injuries. In these cases, some change, such as cerebral edema, may happen in the subcortical region. These delta waves decrease rapidly from 7 to 20 days after the injuries on the injured regions.

And in cases of fresh brain injuries with unconsciousness the delta waves extending over each area of the head, I suppose, may be due to the losing of consciousness.

It is abnormal in epileptic patients that the delta waves (epileptiform activity such as spike waves) appear on the old brain injured regions, and they are present on the opposite and the other regions as well.

But there is an exceptional case, as reported by Jasper, Kershman and Elvidge (1940), in which the delta (spike and seizure waves) waves appear remarkably in spite of the absence of epileptic fit. Such, I suppose, may have relations with the latent convulsive tendency. 


\section{References.}

1) Case, T.J. and Bucy, P.C., J. Neurophysiol., 1938, 1, 245.

2) Jasper, Kershman and Elvidge, Arch. of Neurol. and Psychiat., 1940, 44, 328.

3) Bernhard and Skoglund, Skand. Arch. Physiol., 1939, 82, 178-184.

4) Zeniya, "Nokenkyu" 1948, in press. 